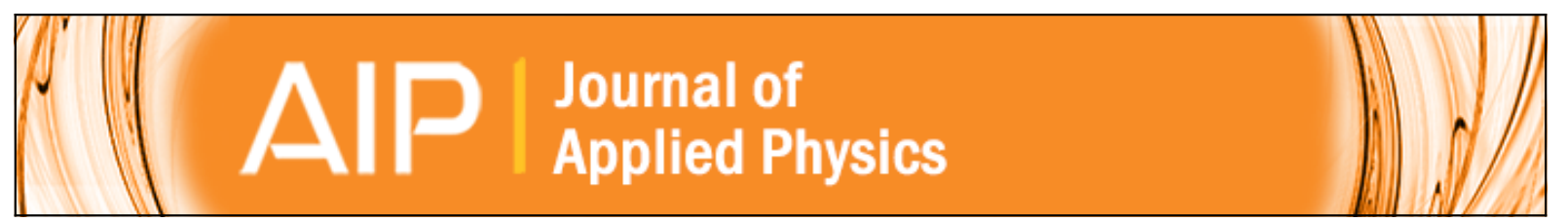

\title{
Formation of dendrite domain structures in stoichiometric lithium niobate at elevated temperatures
}

V. Ya. Shur, D. S. Chezganov, M. S. Nebogatikov, I. S. Baturin, and M. M. Neradovskiy

Citation: Journal of Applied Physics 112, 104113 (2012); doi: 10.1063/1.4766308

View online: http://dx.doi.org/10.1063/1.4766308

View Table of Contents: http://scitation.aip.org/content/aip/journal/jap/112/10?ver=pdfcov

Published by the AIP Publishing

\section{Articles you may be interested in}

Resolution of the ferroelectric domains structure in $(\mathrm{K}, \mathrm{Na}) \mathrm{NbO}$-based lead-free ceramics by confocal Raman microscopy

J. Appl. Phys. 113, 187215 (2013); 10.1063/1.4802096

Fatigue effect in ferroelectric crystals: Growth of the frozen domains

J. Appl. Phys. 111, 124111 (2012); 10.1063/1.4729834

Light-induced superlow electric field for domain reversal in near-stoichiometric magnesium-doped lithium niobate

J. Appl. Phys. 107, 063514 (2010); 10.1063/1.3359721

Phase mapping of domain kinetics in lithium niobate by digital holographic interferometry

J. Appl. Phys. 105, 024106 (2009); 10.1063/1.3068363

Impact of ultraviolet light on coercive field, poling dynamics and poling quality of various lithium niobate crystals from different sources

J. Appl. Phys. 96, 2816 (2004); 10.1063/1.1776324

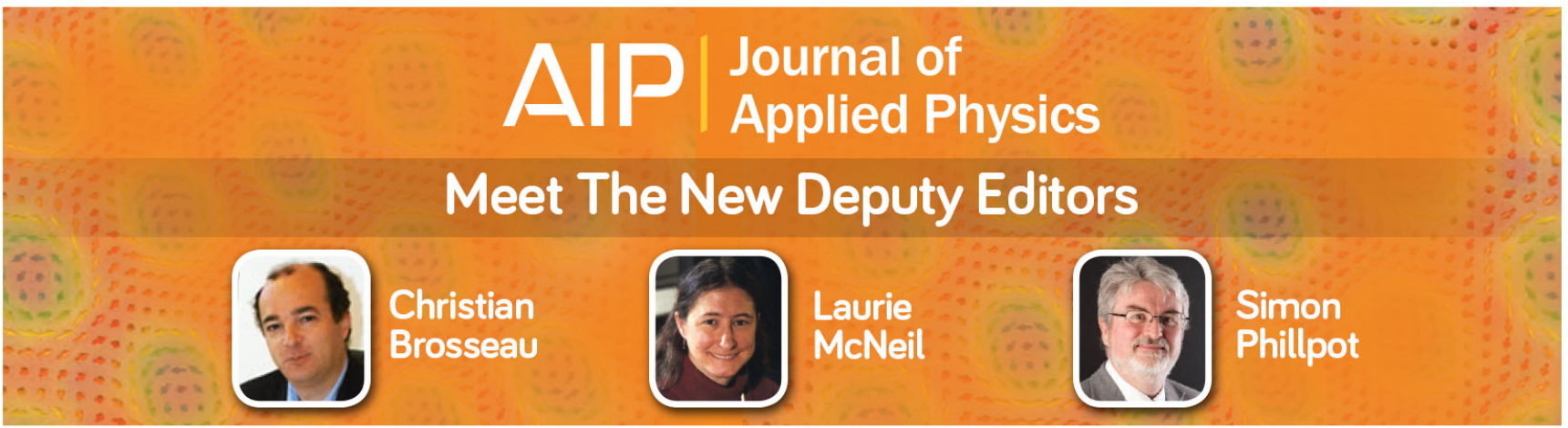




\title{
Formation of dendrite domain structures in stoichiometric lithium niobate at elevated temperatures
}

\author{
V. Ya. Shur, ${ }^{1,2, a)}$ D. S. Chezganov, ${ }^{1}$ M. S. Nebogatikov, ${ }^{1}$ I. S. Baturin,,${ }^{1,2}$ \\ and M. M. Neradovskiy ${ }^{1}$ \\ ${ }^{1}$ Ferroelectric Laboratory, Ural Federal University, 51 Leinin ave, Ekaterinburg, Ekaterinburg 620000 , \\ Russia \\ ${ }^{2}$ Labfer Ltd, 8 Marta street 2-22, Ekaterinburg 620014, Russia
}

(Received 4 September 2012; accepted 20 October 2012; published online 26 November 2012)

\begin{abstract}
Formation of the dendrite-type self-organized domain structures during polarization reversal at elevated temperatures (above $230^{\circ} \mathrm{C}$ ) has been revealed and studied in stoichiometric lithium niobate $\mathrm{LiNbO}_{3}$ single crystals. Optical, confocal Raman, scanning electron, and piezoelectric force microscopy have been used for domain visualization. It has been shown experimentally that formation of the dendrite-like structures has been attributed to correlated nucleation caused by a field distribution in the vicinity of the charged domain walls. (C) 2012 American Institute of Physics. [http://dx.doi.org/10.1063/1.4766308]
\end{abstract}

\section{INTRODUCTION}

Lithium niobate (LN) is one of the most attractive materials for a variety of nonlinear optical and electro-optical applications. Its unique physical properties ${ }^{1}$ inspired the development of various optical devices, including waveguide structures, electro-optical wavelength filters, polarization controllers, efficient nonlinear frequency converters of laser irradiation, ultrafast all-optical signal processing devices, single photon sources, and photonic crystals. ${ }^{2}$ Creation of the tailored regular micro- and nanoscale domain structures (domain engineering) by an electric-field-poling has been confirmed as an established route to improve the optical properties of LN crystals. ${ }^{3,4}$ Needs for lower domain periods $^{5-7}$ and strict fabrication tolerances ${ }^{8}$ stimulate the deep study of the domain structure evolution during polarization reversal. Use of elevated temperature during the electricfiled poling process is also typical, especially for $\mathrm{MgO}$ doped crystals. 5

LN is the uniaxial ferroelectric, which obtains simple domain structure with $180^{\circ}$ domain walls only. Several experimental methods can be applied for the domain visualization with high temporal and spatial resolution. ${ }^{9}$ The screening efficiency and conductivity mechanism can be changed in wide range by crystal heating in ferroelectric phase at temperatures essentially below $\mathrm{T}_{\mathrm{c}}$. All these properties allow using $\mathrm{LN}$ as the model crystal for developing of the domain engineering methods.

\section{A. LN domain shape}

Crucial parameter determining the scenario of the domain kinetics and domain shape is the ratio between switching rate and bulk screening rate. ${ }^{10}$ In the case of "complete screening" (usual experimental conditions) isolated domains in LN demonstrate hexagonal shape with walls strictly oriented along Y crystallographic directions. At the same time,

\footnotetext{
a) Author to whom correspondence should be addressed. Electronic mail: vladimir.shur@usu.ru.
}

the shape of isolated domains essentially changes for ineffective switching conditions. ${ }^{10-12}$ Incomplete screening of the depolarization field hinders the classical domain growth. The experimental study and computer simulation of the domain growth allow obtaining the continuous variation of the isolated domain shape from equilateral hexagon to three-ray stars and ray triplets. ${ }^{11-13}$ Switching process taking place when the screening becomes absolutely ineffective demonstrates qualitatively new scenarios of domain structure evolution and various exotic discrete mechanisms of domain growth. As a result various self-organized structures, ${ }^{10,13-15}$ such as long nanoscale domain arrays, develop. The formation of such domain structures has been attributed to the correlated nucleation effect.

\section{B. Properties of stoichiometric lithium niobate}

The most common LN crystals grown by Czochralski technique have congruent composition (CLN) with a deviation from the stoichiometry $([\mathrm{Li}] /[\mathrm{Nb}]=0.942),{ }^{16,17}$ which leads to the presence of such defects as niobium antisites $\left(\mathrm{Nb}_{\mathrm{Li}}\right)^{4+}$ and lithium vacancies $\left(\mathrm{V}_{\mathrm{Li}}\right)^{-} \cdot{ }^{17}$ The defects have an adverse effect on the crystal properties. It is well known that LN with almost stoichiometric composition (SLN), ${ }^{18,19}$ i.e., with $\mathrm{Li}_{2} \mathrm{O}$ content approaching $50.0 \mathrm{~mol}$. \%, shows an increase of the electro-optical coefficient $r_{33}$, which could have an important impact on electro-optical modulators technology, and an enhancement of the photorefractive effect for holographic memories. ${ }^{20,21}$ An internal field of about $2.5 \mathrm{kV} /$ mm observed in CLN crystals almost disappeared in SLN. ${ }^{22}$ The values of the ferroelectric threshold field tend to decrease in a dramatic way as the $\mathrm{Li} / \mathrm{Nb}$ ratio is approaching unit. $^{22-24}$ The lower threshold field in SLN facilitates the fabrication of the tailored domain structures.

\section{Elevated temperature}

The electronic type of conductivity dominates in all crystals of LN family at room temperature. The conductivity increases during heating and at the temperature above $170^{\circ} \mathrm{C}$ 

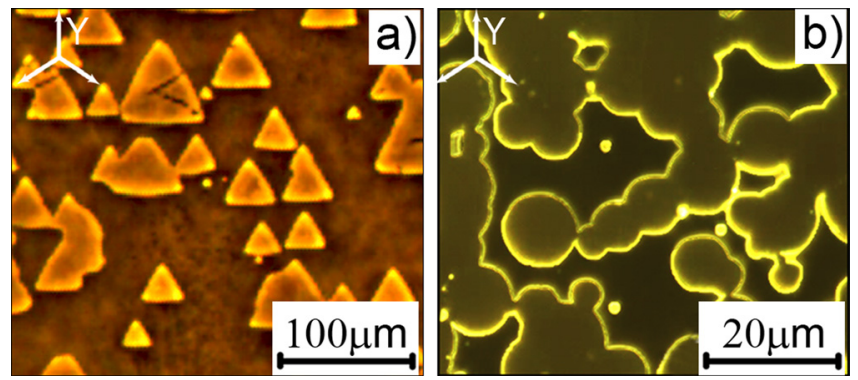

FIG. 1. Optical images of CLT domains revealed by selective chemical etching. Switching at different temperatures: (a) $125^{\circ} \mathrm{C}$, (b) $250^{\circ} \mathrm{C}$.

the ionic type dominates. ${ }^{25}$ It has been proposed that electronic conductivity along Y polar axis is anisotropic. ${ }^{26}$ This assumption has been used for explanation of the anisotropic growth of elementary steps along the domain wall, which leads to formation of the plane walls, determined nucleation and growth of hexagonal domains. ${ }^{26}$ This nucleation mechanism appeared itself in the domain shape stability effect representing recovery of the hexagonal shape after merging of isolated domains. The triangular domain shape is obtained in congruent lithium tantalate (CLT) (Fig. 1(a)).

The determined nucleation for LN and LT has changed to stochastic one at elevated temperatures where ionic conductivity prevails. The growth of the irregular shaped and circular domains has been observed in CLT (Fig. 1(b)). The revealed lack of the domain shape stability has led to formation of the complicated domain shapes including selforganized dendrite- type domain structures. ${ }^{10,13}$

\section{Dendrite domain structures in ferroelectrics}

The randomly oriented dendrite domain patterns were obtained experimentally in $\mathrm{Pb}\left(\mathrm{Zn}_{1 / 3} \mathrm{Nb}_{2 / 3}\right) \mathrm{O}_{3}-\mathrm{PbTiO}_{3}(\mathrm{PZN}-$ PT) [001] single crystals after application of the electric field pulses. $^{27}$ It was suggested that these unusual domain structures were a result of local defects and space charge in the relaxor PZN-PT crystals.

Formation of dendrite domain structures as a result of spontaneous backswitching after fast removal of the external field was observed during periodical poling of CLN in the regions between the stripe electrodes. ${ }^{10,11}$ It was shown also that the dendrite structure was appeared during backswitching as a result of finger growth from the boundaries of the hexagonal domains. This effect was attributed to instability of the domain wall shape under highly non-equilibrium switching conditions induced by super-strong backswitching fields. 10,13

The present paper is devoted to study of the formation of dendrite-like domain shapes in the stoichiometric lithium niobate crystals during polarization reversal at the elevated temperatures.

\section{EXPERIMENT}

The domain shape evolution during switching at elevated temperatures was studied in $\mathrm{Z}^{-}$cut optical grade $0.5 \mathrm{~mm}$ thick $\mathrm{LiNbO}_{3}$ single crystalline wafers with quasistoichiometric composition, grown by Czochralski method adding $\mathrm{K}_{2} \mathrm{O}$ during the growth process by SAES Getters S.P.A., Italy. ${ }^{28}$ The sputtered 50 -nm-thick $\mathrm{Cr}$ or $\mathrm{In}_{2} \mathrm{O}_{3}: \mathrm{Sn}$ conductive oxide (ITO) were used as the circular shape electrodes with a diameter of $2-3 \mathrm{~mm}$ on $\mathrm{Z}^{+}$polar surface and solid electrodes on $\mathrm{Z}^{-}$surface.

The polarization reversal was made by application of the single field pulse or several pulses using high voltage amplifier TREK Model 20/20c. The asymmetric triangle field pulse waveform was used (Fig. 2). The field increased with rate of $500 \mathrm{~V} / \mathrm{s}$ up to field maximum $\mathrm{E}_{\max }$ in the ranges of $1.10-1.65 \mathrm{kV} / \mathrm{mm}$ and decreased to zero during $0.5 \mathrm{~s}$. The sample was clamped between spring-loaded aluminum rod and copper plate. The rod diameter was smaller than that of the deposited electrode. Such assembly was immersed in a silicon oil bath with an electrical heater driven by a PID temperature controller Owen TPM151. Before polarization reversal the sample was heated slowly up to 250 and $275^{\circ} \mathrm{C}$.

The static domain structures formed after partial polarization reversal were visualized by four methods after chemical removal of the electrodes.

1. The piezoresponse force microscopy (PFM) represents the nondestructive method which allows visualizing the domain structure at the surface with high spatial resolution. ${ }^{29}$ A scanning probe microscope NTEGRA Aura (NT MDT, Russia) having silicon DCP20 tips with a diamondlike conductive coating and a typical radius of the curvature about $50 \mathrm{~nm}$ (NT-MDT, Russia) was used. AC excitation voltage $\mathrm{U}_{\text {mod }}$ with amplitude $5 \mathrm{~V}$ and frequency 17.4 $\mathrm{kHz}$ was applied between the conductive tip and the solid bottom electrode in order to induce the piezoelectric response. A signal of vertical cantilever deflection, proportional to the piezoelectric surface oscillation was analyzed by lock-in amplifier SR-830 (Stanford Research Systems, USA). The obtained amplitude and phase of the PFM signal provided information about the domain structure on the surface. ${ }^{30,31}$ The estimated lateral resolution was about $50 \mathrm{~nm}$.

2. The Raman confocal microscopy (RCM) is a new method for the domain visualization combining analytical abilities of a Raman spectroscopy and a high spatial resolution (below the diffraction limit), inherent to the confocal microscopy. It has been applied recently for domain visualization in LN and LT crystals both at the surface and in the bulk. ${ }^{32-34}$ It has been shown in LN that mechanical stress

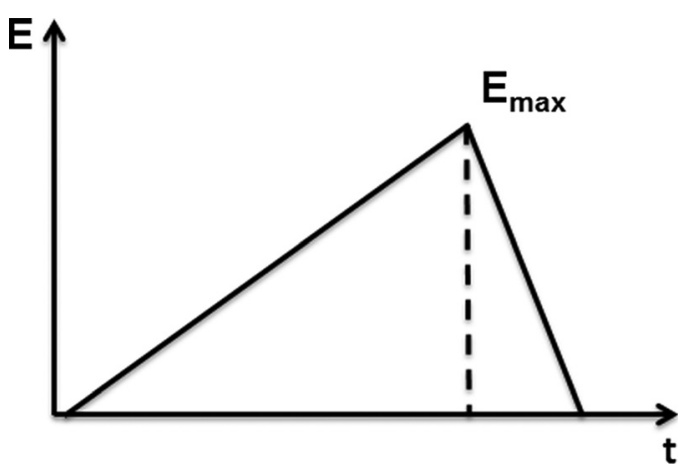

FIG. 2. Field pulse waveform. 
induced by electric field in the vicinity of domain walls leads to changes of the certain lines of the Raman spectrum. By analysis of intensity and position of these lines during 3D scanning we have obtained the set of 2D images at different depths with a sufficient contrast of the domain walls. RCM imaging has been done using NTEGRA Spectra (NT MDT, Russia). The Raman spectra were recorded in $\mathrm{Z}(\mathrm{XX}) \mathrm{Z}$ configuration at room temperature using $\mathrm{He}-$ Ne laser $(633 \mathrm{~nm}, 30 \mathrm{~mW})$ as a pumping source. Exposure time of $0.2-0.77 \mathrm{~s}$ and confocal pinhole diameter of $75 \mu \mathrm{m}$ were used. The estimated RCM lateral resolution was about $300 \mathrm{~nm}$ and about $500 \mathrm{~nm}$ in $\mathrm{Z}$ direction.

3. The optical microscopy (Olympus BX-51, Japan) in transmitted and dark field modes was used for visualization of a surface relief revealed by selective chemical etching by pure hydrofluoric acid (HF) at room temperature for $1.5-2.5 \mathrm{~min}$. The estimated lateral resolution was about $300 \mathrm{~nm}$.

4. CrossBeam Workstation AURIGA (Carl Zeiss NTS, Germany) was used for the visualization of the etched surface relief with the highest spatial resolution. The workstation was equipped by Carl Zeiss NTS' charge compensation system, which was used for local charge compensation by gaseous nitrogen to minimize a surface charging by electrons. The SE and In-Lens detector were used for imaging. The obtained highest lateral resolution about $2 \mathrm{~nm}$ was achieved for $4 \mathrm{kV}$.

\section{RESULTS}

Appearance of the dendrite domains was observed for switching at the elevated temperatures above $230{ }^{\circ} \mathrm{C}$ (Fig. 3). After partial switching the isolated domains with typical sizes ranged from 3 to $30 \mu \mathrm{m}$ were distributed over the whole $\mathrm{Z}^{+}$polar surface covered by electrode. The total shape of the domain ensembles was close to six-ray star with vertices oriented strictly along Y crystallographic direction (Figs. 3(a)3(c)). It should be pointed out that the average length of $\mathrm{Y}^{+}$ oriented rays is longer than for $\mathrm{Y}^{-}$ones. This effect can be attributed to nonequivalence of $\mathrm{Y}^{+}$and $\mathrm{Y}^{-}$crystallographic directions of $Y$ polar axis. The domain walls of the branches exhibited irregular shape (loss of the domain shape stability effect) during formation of the new generation branches (Figs. 3(b) and 3(c)). This effect enhanced essentially for application of the several electric field pulses leading to formation of more complicated dendrite structures (Figs. 3(c) and 3(d)).

An application of the single field pulse led to formation of the six-ray star domain shapes (Figs. 3(a) and 3(b)). Such domains were obtained on the $\mathrm{Z}^{+}$surface over the whole electrode area. The absence of the domain images revealed on $\mathrm{Z}^{-}$surface indicated that all domains had charged domain walls. The appearance of new domains occurred during the whole switching process in linear increasing field, thus, domains of various shapes and sizes were obtained after single pulse (Fig. 4). The observed domain structures can be considered as corresponding to the different stages of the domain growth and can be attributed to continuous nucleation as a result of nonuniform switching for single pulse. It was
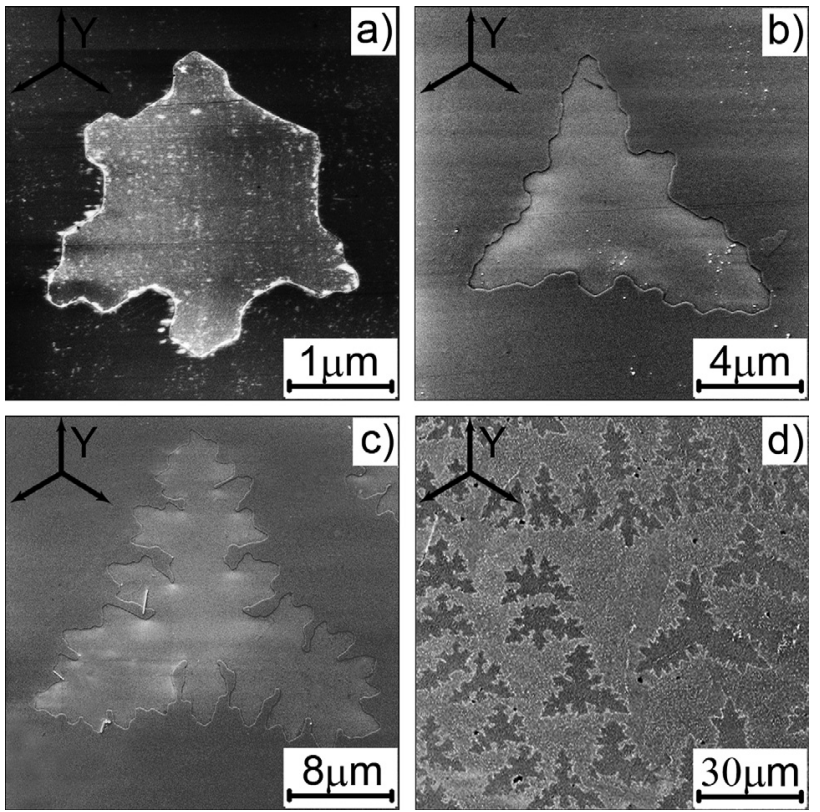

FIG. 3. SEM images of the dendrite domain structures with different magnification revealed by selective chemical etching $\mathrm{T}=250^{\circ} \mathrm{C}$.

shown that various shapes of isolated domains have been appeared in different fields. Hexagon shape corresponded to "young" domains formed in high field, star shape-to "middle-age" domains appeared in average field, and irregular shape - to "old" ones appeared in low field.

These domain structures qualitatively coincided with domain shapes obtained for various $\mathrm{E}_{\max }$ (Fig. 5).

The domain structure evolution of nonthrough domains appeared at the polar surface has been reconstructed by analysis of the set of RCM domain images obtained at different depths. ${ }^{33}$ The analysis was based on the following assumptions: (1) the domains nucleated at the polar surface only, (2) the ratio of the domain growth in lateral and vertical direction was constant. These assumptions were proved to be reasonable for explanation of the experimental data. ${ }^{33}$ In such conditions the deeper images corresponded to the earlier stages of the domain evolution at the polar surface: "the deeper - the earlier".

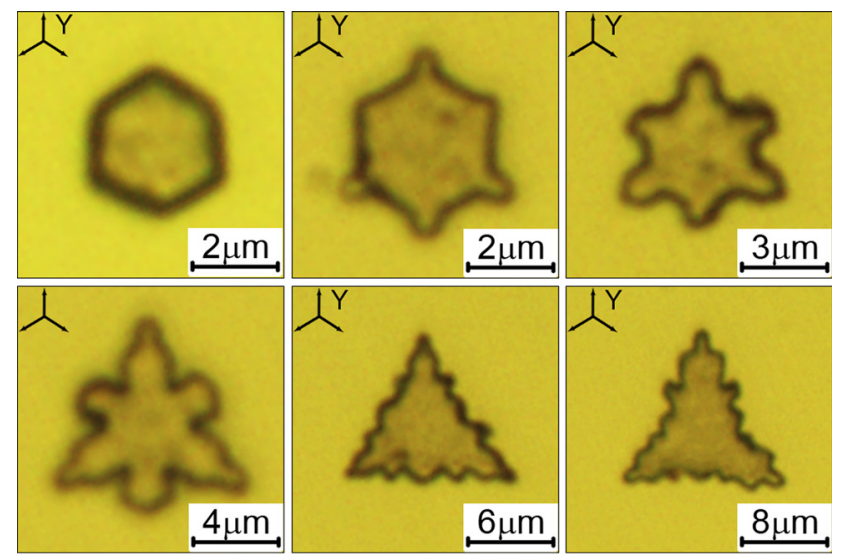

FIG. 4. Optical images of the dendrite structure evolution during single pulse $\mathrm{E}_{\max }=1.5 \mathrm{kV} / \mathrm{mm}$. 

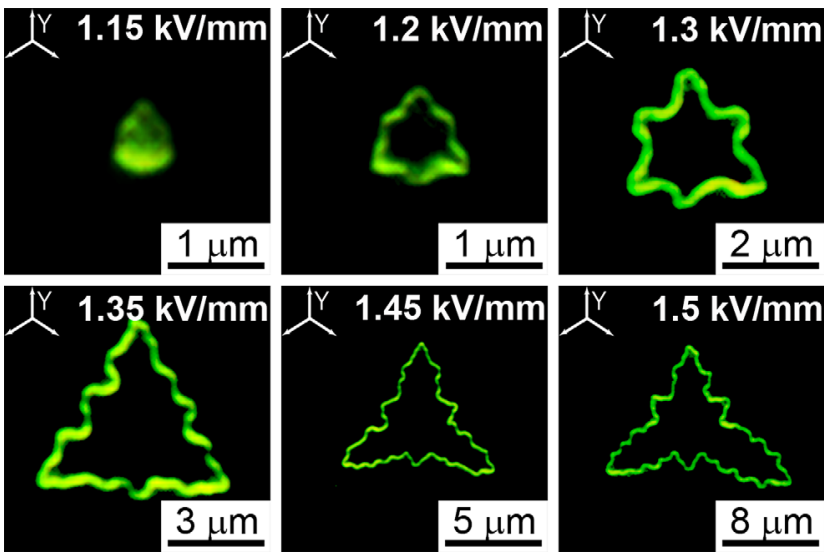

FIG. 5. Optical images (dark field) of the domain shape dependence on $E_{\max }$ for single pulse.

The analysis allowed us to reveal two mechanisms of the dendrite domain structure formation during application of the single pulse corresponding to different field ranges (Fig. 6). The discrete switching (correlated formation of the isolated domains near existing domains ${ }^{13,26}$ ) and subsequent domain merging was obtained at low fields (Figs. 6(a)-6(c)). The continuous domain growth was obtained at high field (Figs. 6(d)-6(f)). The obtained unsymmetrical domain shape is due to the interaction of the growing domain with the neighboring one.

The following stages of the domain evolution during application of the single pulse were revealed (Fig. 7). (i) Appearance of a single isolated domain. (ii) Appearance of three isolated domains at equal distance from the central domain in $\mathrm{Y}^{+}$directions which suppressed a further growth of the central one. (iii) Appearance of three isolated domains at equal distance from the central domain in $\mathrm{Y}^{-}$directions. (iv) Oriented growth of the domain chains with a sub-micron period in $\mathrm{Y}^{+}$directions. (v) Formation of six-ray stars as a result of domain merging. (vi) Formation of star-like structure as a result of ray broadening.

The application of two consecutive pulses leads to the formation of the dendrite shape domains as it is shown at Figs. 8(a) and 8(b). The dendrite shape became even more complex after three pulses (Fig. 8(c)).

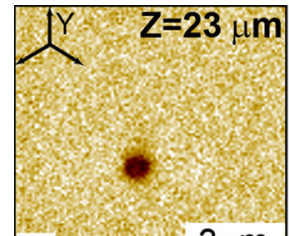

a)

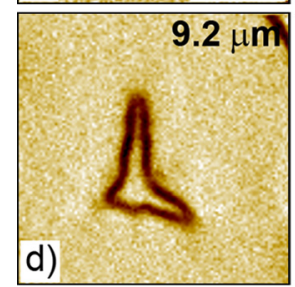

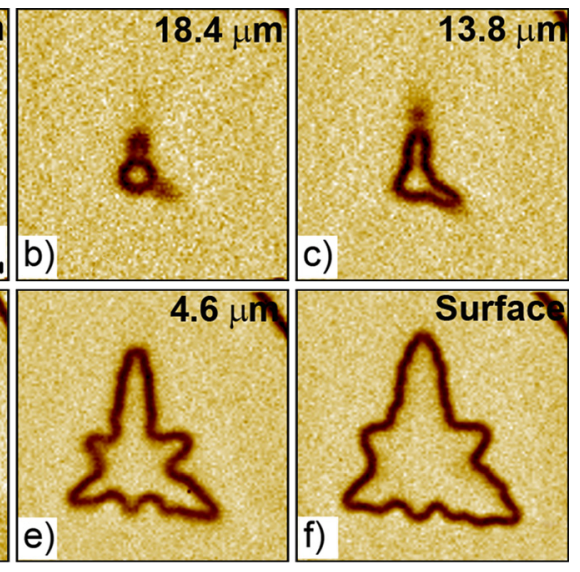

FIG. 6. Domain structure evolution during application of the single field pulse extracted from the confocal images at the different depth: (a)-(c) discrete switching, (d)-(f) continuous switching.
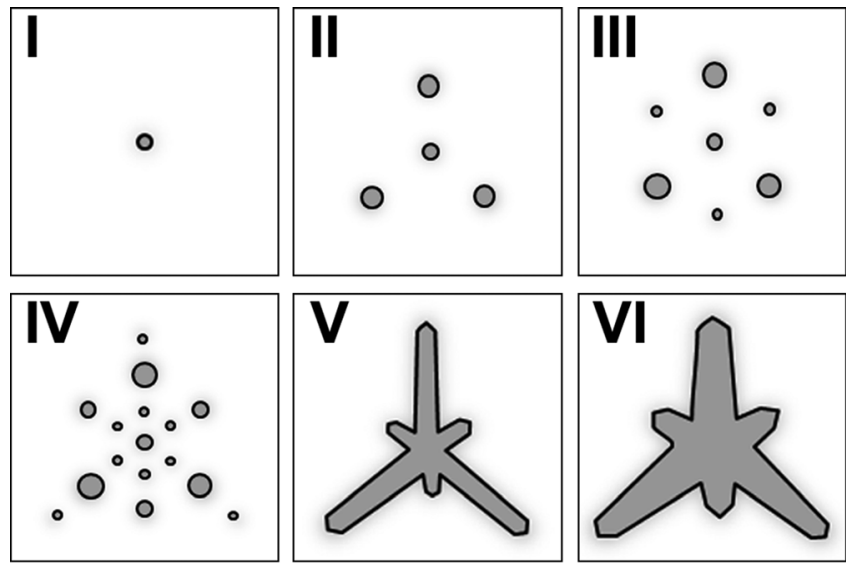

FIG. 7. Scheme of the dendrite domain structure evolution during application of the single field pulse.

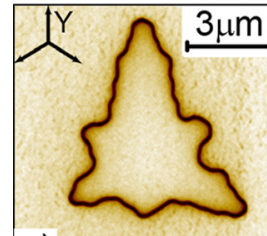

a)

FIG. 8. PFM image of dendrite structures for various number of field pulses: (a) single pulse $\mathrm{E}_{\max }=1.6 \mathrm{kV} / \mathrm{mm}$, (b) two pulses $\mathrm{E}_{\max }=1.6 \mathrm{kV} / \mathrm{mm}$, (c) three pulses. $\mathrm{E}_{\max }=1.5 \mathrm{kV} / \mathrm{mm}$.

The analysis of RCM domain images obtained after two pulses (Fig. 9) allowed us to reveal the main stages of the domain formation during the second pulse (Fig. 10). (1) Appearance of six isolated domains of second generation near the ray ends. (2) Correlated formation of 15 isolated domains of the second generation around six previous domains which suppressed the further ray elongation. The domains at $\mathrm{Y}^{+}$ray ends generated three new domains, while the domains at $\mathrm{Y}^{-}$ends generated two new domains, because one direction was already occupied by domain ray formed during the first pulse. (3) Merging of the isolated domains of
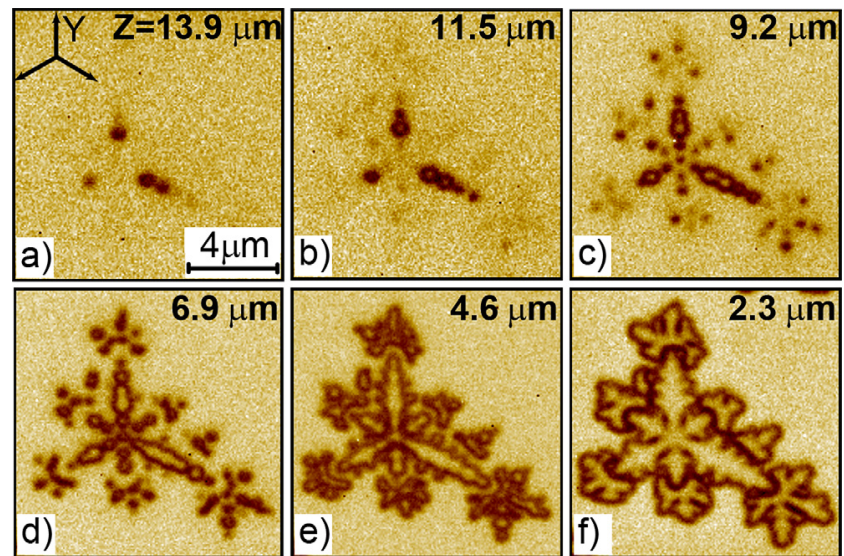

FIG. 9. The formation of the domain structures during application of two pulses extracted from the RCM images obtained at the different depths: (a)-(c) hexagonal domain star during the first pulse, (d)-(f) dendrite domain structure during the second pulse. 

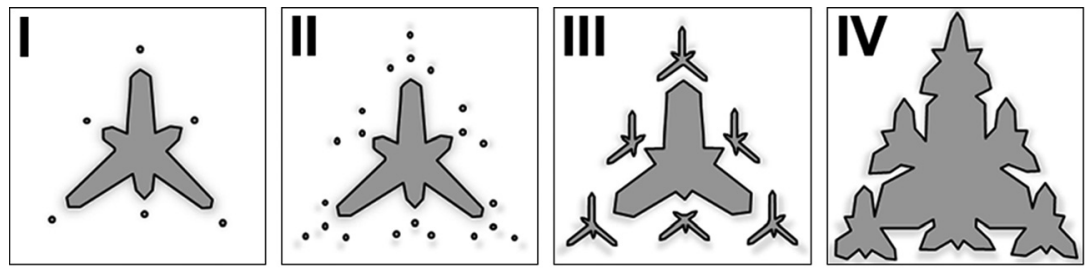

FIG. 10. Scheme of the main stages of dendrite domain structure formation during application of the second pulse.

the second generation into five-ray stars. (4) Growth of the star domains by sideways wall motion and their merging. The incomplete merging process led to existence of the residual nanodomains which resulted in a RCM contrast between old and new domains. The dendrite domain evolution during application of the third pulse occurred in the similar manner.

The statistical analysis of the domain images allowed us to reveal the following periods of the structures: (1) main period at the first and the second stages for the single pulse $1250 \pm 20 \mathrm{~nm}$; (2) correlated nucleation period $580 \pm 50 \mathrm{~nm}$; (3) correlated nucleation period at the second pulse $450 \pm 100 \mathrm{~nm}$.

It is necessary to point out that at the final stage of the dendrite structure formation the lack of the wall shape stability was observed. The nanodomains were formed at a given distance from the moving walls of the initial domain rays. The short branches of the second generation (fingers) oriented along $\mathrm{Y}$ direction were formed as a result of nanodomain growth and merging with the initial rays. The effect was more pronounced for switching at higher temperatures (Fig. 11).

\section{DISCUSSION}

The obtained experimental facts allowed us to suggest the self-organized formation mechanism of the dendrite domain structures. It was shown that the framework of the dendrite structure appeared due to formation of isolated domain and subsequent oriented growth of the nanodomain chains (discrete switching).

The nanodomain period in the chains was defined by correlated nucleation effect. ${ }^{13,26}$ It was shown by computer simulation that the period of the chain of isolated needle-like domains with charged domain walls was defined by the length of the last domain in the chain. ${ }^{35}$

The anisotropic chain growth strictly along Y crystallographic directions observed experimentally in all crystals of
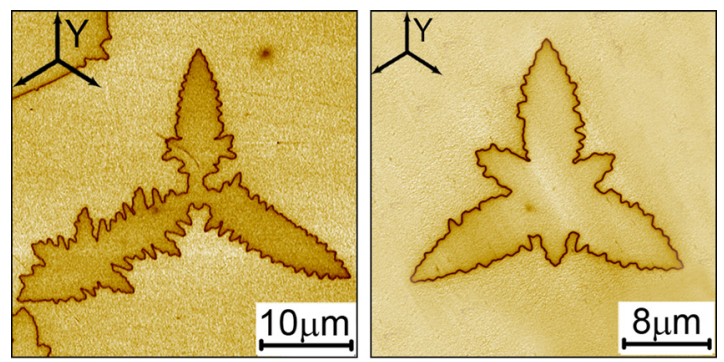

FIG. 11. PFM mages of dendrite domain structure. $\mathrm{T}=275^{\circ} \mathrm{C}$. Single pulse $\mathrm{E}_{\max }=1.4 \mathrm{kv} / \mathrm{mm}$.
LN family can be attributed to anisotropy of the bulk screening mechanism. ${ }^{26}$

The switching at the elevated temperatures stimulated the stochastic nucleation instead of the determined nucleation at low temperatures. This fact led to qualitative change of the domain shape. The determined generation of the steps (position of the nucleation sites) at the vertices of the polygon-shape domain at low temperatures resulted in the effect of domain shape stability after domain merging. ${ }^{12,36}$ For instance, hexagon domain with $\mathrm{Y}$ oriented domain walls was formed after merging of two hexagon domains. ${ }^{11,12,36}$ The independent domain growth after merging in the case of stochastic nucleation resulted in formation of complicated domain shapes including dendrite-like domain structures. The substitution of the dominated nucleation mechanism at the elevated temperature can be attributed to change of the mechanism of electrical conductivity from anisotropic electronic to isotropic ionic one.

The formation of the residual domain chains during merging of the enlarged neighboring domains was caused by electrostatic interaction between approaching domain walls. The residual depolarization fields appeared after moving domain wall for incomplete switching hampered the nucleation at the domain wall and terminated the coming wall motion. ${ }^{10,13}$

\section{CONCLUSION}

We have demonstrated the formation of dendrite domain structures during polarization reversal at elevated temperature (above $230^{\circ} \mathrm{C}$ ) in single crystals of stoichiometric lithium niobate. It has been shown experimentally that the obtained formation of the self-organized dendrite domain structures has been attributed to correlated nucleation caused by field distribution in the vicinity of the charged domain walls. It has been supposed that formation of the complicated domain shapes is caused by the stochastic nucleation at the elevated temperatures instead of determined nucleation at low temperatures due to change of the mechanism of electrical conductivity from anisotropic electronic to isotropic ionic one.

\section{ACKNOWLEDGMENTS}

The equipment of the Ural Center for Shared Use "Modern Nanotechnology", Institute of Natural Sciences of the Ural Federal University has been used. The research was made possible in part by RFBR (Grant Nos. 10-02-96042-rUral-a, 10-02-00627-a, and 11-02-91066-CNRS-a), by Ministry of Education and Science (Contract 16.55211 7020), by OPTEC LLC and by the financial support of young 
scientists of the Ural Federal University development program.

${ }^{1}$ R. S. Weis and T. K. Gaylord, Appl. Phys. A 37, 191 (1985).

${ }^{2}$ W. Sohler, H. Hu, R. Ricken, V. Quiring, C. Vannahme, H. Herrmann, D. Buchter, S. Reza, W. Grundkotter, S. Orlov, H. Suche, R. Nouroozi, and Y. Min, Opt. Photonics News 19, 24 (2008).

${ }^{3}$ R. L. Byer, J. Nonlinear Opt. Phys. Mater. 6, 549 (1997).

${ }^{4}$ D. Hum and M. Fejer, C. R. Phys. 8, 180 (2007).

${ }^{5}$ K. Mizuuchi, A. Morikawa, T. Sugita, and K. Yamamoto, J. Appl. Phys. 96, 6585 (2004).

${ }^{6}$ V. Ya. Shur, E. L. Rumyantsev, E. V. Nikolaeva, E. I. Shishkin, D. V. Fursov, R. G. Batchko, L. A. Eyres, M. M. Fejer, and R. L. Byer, Appl. Phys. Lett. 76, 143 (2000).

${ }^{7}$ C. Canalias and V. Pasiskevicius, Nat. Photonics 1, 459-462 (2007).

${ }^{8}$ M. M. Fejer, G. A. Magel, D. H. Jundt, and R. L. Byer, IEEE J. Quantum Electron. 28, 2631 (1992).

${ }^{9}$ E. Soergel, Appl. Phys. B 81, 729 (2005).

${ }^{10} \mathrm{~V}$. Ya. Shur, in Nucleation Theory and Applications, edited by J. W. P. Schmelzer (Wiley-VCH Verlag GmbH \& Co. KGaA, Weinheim, 2005).

${ }^{11}$ V. Ya. Shur, A. I. Lobov, A. G. Shur, E. L. Rumyantsev, and K. Gallo, Ferroelectrics 360, 111 (2007).

${ }^{12}$ A. I. Lobov, V. Ya. Shur, I. S. Baturin, E. I. Shishkin, D. K. Kuznetsov, A. G. Shur, M. A. Dolbilov, and K. Gallo, Ferroelectrics 341, 109 (2006).

${ }^{13}$ V. Ya. Shur, J. Mater. Sci. 41, 199 (2006).

${ }^{14}$ V. Ya. Shur, E. L. Rumyantsev, A. G. Shur, A. I. Lobov, D. K. Kuznetsov, E. I. Shishkin, E. V. Nikolaeva, M. A. Dolbilov, P. S. Zelenovskiy, K. Gallo, and M. P. De Micheli, Ferroelectrics 354, 145 (2007).

${ }^{15}$ V. Ya. Shur, D. K. Kuznetsov, E. A. Mingaliev, E. M. Yakunina, A. I. Lobov, and A. V. Ievlev, Appl. Phys. Lett. 99, 082901 (2011).

${ }^{16}$ K. Kitamura, J. K. Yamamoto, N. Iyi, S. Kirnura, and T. Hayashi, J. Cryst. Growth 116, 327 (1992).

${ }^{17}$ N. Iyi, K. Kitamura, F. Izumi, J. K. Yamamoto, T. Hayashi, H. Asano, and S. Kimura, J. Solid State Chem. 101, 340 (1992).

${ }^{18}$ T. Fujiwara, M. Takahashi, M. Ohama, A. J. Ikushima, Y. Furukawa, and K. Kitamura, Electron. Lett. 35, 499 (1999).
${ }^{19}$ F. Abdi, M. Aillerie, K. Chan, P. Bourson, M. D. Fontana, and K. Polgar, J. Appl. Phys. 84, 2251 (1998).

${ }^{20}$ Y. Kondo, T. Fukuda, Y. Yamashita, K. Yokoyama, K. Arita, M. Watanabe, Y. Furukawa, K. Kitamura, and H. Nakajima, Jpn. J. Appl. Phys., Part 1 39, 1477 (2000).

${ }^{21}$ Y. Furukawa, K. Kitamura, S. Takekawa, A. Miyamoto, M. Terao, and N. Suda, Appl. Phys. Lett. 77, 2494 (2000).

${ }^{22}$ V. Gopalan, T. E. Mitchell, Y. Furukawa, and K. Kitamura, Appl. Phys. Lett. 72, 1981 (1998).

${ }^{23}$ A. Grisard, E. Lallier, K. Polgar, and A. Peter, Electron. Lett. 36, 1043 (2000).

${ }^{24}$ V. Ya. Shur, E. V. Nikolaeva, E. I. Shishkin, V. L. Kozhevnikov, A. P. Chernykh, K. Terabe, and K. Kitamura, Appl. Phys. Lett. 79, 3146 (2001).

${ }^{25}$ K. K. Wong, Properties of Lithium Niobate (The Institution of Engineering and Technology, 2002), pp. 91-96.

${ }^{26}$ V. Ya. Shur, in Advanced Dielectric, Piezoelectric, and Ferroelectric Materials-Synthesis, Characterization \& Applications, edited by Z. G. Ye (Woodhead Publishing Ltd., Cambridge, 2008), p. 622.

${ }^{27}$ H. Yu and C. A. Randall, J. Appl. Phys. 86, 5733 (1999).

${ }^{28}$ F. Caccavale, D. Callejo, C. Dragoni, A. Morbiato, M. Musolino, and M. Properzi, Proc. SPIE 5621, 77 (2004).

${ }^{29}$ A. Gruverman and S. V. Kalinin, J. Mater. Sci. 41, 107 (2006).

${ }^{30}$ E. I. Shishkin, A. V. Ievlev, E. V. Nikolaeva, M. S. Nebogatikov, and V. Ya. Shur, Ferroelectrics 374, 26 (2008).

${ }^{31}$ V. Ya. Shur, A. V. Ievlev, E. V. Nikolaeva, E. I. Shishkin, and M. M. Neradovskiy, J. Appl. Phys. 110, 052017 (2011).

${ }^{32}$ P. Zelenovskiy, V. Ya. Shur, P. Bourson, M. D. Fontana, D. K. Kuznetsov, and E. A. Mingaliev, Ferroelectrics 398, 34 (2010).

${ }^{33}$ P. Zelenovskiy, M. Fontana, V. Shur, P. Bourson, and D. Kuznetsov, Appl. Phys. A 99, 741 (2010).

${ }^{34}$ V. Ya. Shur, P. S. Zelenovskiy, M. S. Nebogatikov, D. O. Alikin, M. F. Sarmanova, A. V. Ievlev, E. A. Mingaliev, and D. K. Kuznetsov, J. Appl. Phys. 110, 052013 (2011).

${ }^{35}$ V. Ya. Shur, A. I. Lobov, E. L. Rumyantsev, and D. K. Kuznetsov, Ferroelectrics 399, 68 (2010).

${ }^{36}$ I. S. Baturin, M. V. Konev, A. R. Akhmatkhanov, A. I. Lobov, and V. Ya. Shur, Ferroelectrics 374, 136 (2008). 